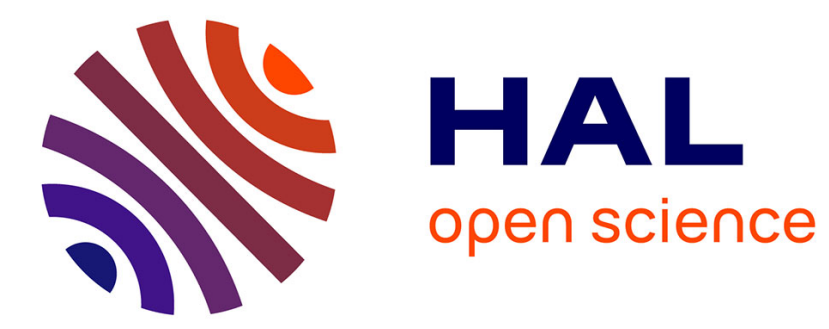

\title{
Prurigos chroniques et leur exploration
}

A. Du-Thanh

\section{To cite this version:}

A. Du-Thanh. Prurigos chroniques et leur exploration. Revue francaise d'allergologie, 2019, 59, pp.133

- 135. 10.1016/j.reval.2019.02.013 . hal-03486319

\section{HAL Id: hal-03486319 \\ https://hal.science/hal-03486319}

Submitted on 20 Dec 2021

HAL is a multi-disciplinary open access archive for the deposit and dissemination of scientific research documents, whether they are published or not. The documents may come from teaching and research institutions in France or abroad, or from public or private research centers.
L'archive ouverte pluridisciplinaire HAL, est destinée au dépôt et à la diffusion de documents scientifiques de niveau recherche, publiés ou non, émanant des établissements d'enseignement et de recherche français ou étrangers, des laboratoires publics ou privés.

\section{다)(1) $(5$}

Distributed under a Creative Commons Attribution - NonCommercial| 4.0 International 
Version of Record: https://www.sciencedirect.com/science/article/pii/S1877032019300326

Manuscript_6c4ab65ea3ba9b82b487a74b3e3cc58a

\title{
Prurigos chroniques et leur exploration
}

Chronic prurigo: definitions and investigations

\author{
Aurélie Du-Thanh* \\ CHU de Montpellier \\ Département de Dermatologie \\ Hôpital Saint-Eloi, 80 avenue Augustin Fliche, 34295 Montpellier \\ Téléphone 0467337580 \\ Fax 0467336958 \\ Email : a-du thanh@chu-montpellier.fr
}


Mots-clés : prurigo, prurit

Keywords: prurigo, pruritus

\section{II n'existe pas de définition satisfaisante du terme « prurigo ».}

II désigne des lésions cutanées clairement entretenues sinon déclenchées par le grattage. Les dermatoses prurigineuses constituent l'essentiel de la Dermatologie dite inflammatoire, et concernent aussi certaines oncodermatoses comme les lymphomes cutanés $T$ épidermotropes. Le terme " prurigo » recouvre plusieurs entités qui partagent un aspect de dermatose prurigineuse papuleuse et excoriée. On distingue les prurigos aigus, le plus souvent ectoparasitaires (post-piqûre) et les prurigos chroniques.

En 2017, dans le cadre d'un projet d'étude en cours sur le prurigo chronique, un groupe d'experts européens a proposé la définition suivante : « présence d'un prurit chronique depuis au moins 6 semaines, avec des lésions cutanées multiples localisées ou généralisées, excoriées, sous forme de papules rosées, de nodules et/ou de plaques, survenant dans le cadre d'un cycle entre prurit et lésions de grattage, et liées à une sensibilisation neuronale » [1].

Certaines entités ont des caractéristiques particulières et spécifiques : prurigo gravidique, prurigo actinique, prurigo pigmentosa.

Les examens paracliniques indiqués dans l'exploration d'un prurigo dépendront de l'anamnèse et sont principalement orientés vers la recherche d'une néoplasie sous-jacente, par analogie avec le bilan de tout prurit généralisé sine materia.

\section{Le prurigo nodulaire (de Hyde)}

Dans le prurigo nodulaire de Hyde, forme emblématique des prurigos chroniques, les lésions papulo-nodulaires centimétriques violacées sont excoriées, croûteuses, ou pigmentées au stade séquellaire. La topographie des lésions est plutôt symétrique, épargne typiquement les zones inaccessibles au grattage, au milieu du dos mais également la région palmoplantaire et les muqueuses, alors qu'elles prédominent sur les faces externes des cuisses et des bras, les fesses et peuvent atteindre le visage [2].

Son incidence n'est pas connue, mais la majorité des cas concerneraient des femmes de plus de 40 ans.

L'histopathologie du prurigo nodulaire de Hyde n'est pas spécifique Elle retrouve une hyperkératose orthokératosique compacte, moins de spongiose et davantage de fibrose dermique que dans les prurigos aigus, ainsi que des lymphocytes, des macrophages, des mastocytes et des polynucléaires neutrophiles aux côtés des polynucléaires éosinophiles dans l'infiltrat dermique.

Les mécanismes physiopathologiques du prurigo chronique ne sont pas complètement connus

Cependant la plupart des auteurs évoquent une hypothèse neurocutanée, avec des fibres nerveuses en nombre augmenté mais hypoplasiques dans le derme superficiel, une surexpression du NGF (nerve growth factor) et de son récepteur notamment mastocytaire, ainsi que de plusieurs neuropeptides tels que la substance $P$ et son récepteur NK1R [3]. Par ailleurs, la sur-représentation de cytokines de type Th2 dans les lésions cutanées par rapport à la peau apparemment saine adjacente plaide pour leur implication. 


\section{Le prurigo de Besnier}

II désigne, chez certains patients souffrant de dermatite atopique, des lésions de prurigo associées à leurs plaques habituelles d'eczéma. Cette présentation particulière peut être due à un vrai prurigo aigu ectoparasitaire (on parle alors de prurigo de Hébra) ou à des lésions chroniques pouvant correspondre à un endo(phéno)type de la dermatite atopique au même titre que les formes à type d'eczéma nummulaire [4].

\section{Le prurigo gravidique}

Il appartient au spectre désormais nommé " éruption polymorphe de la grossesse » (EPG), autrefois désigné sous le terme de PUPP pour « Pruritic urticarial papules and plaques of pregnancy ". L'incidence de l'EPG est de 1/160 à 300 grossesses, elle débute classiquement au niveau des vergetures, dans $3 / 4$ des cas à la fin de la première grossesse et/ou en cas de grossesse gémellaire, et régresse après l'accouchement, sans conséquence particulière pour la mère ou l'enfant. L'aspect réalisé est variable : pseudo-urticarien ou à type de prurigo avec une distribution diffuse possible [5].

Sa physiopathologie n'est pas connue, elle pourrait relever d'une réaction immunoallergique à des cellules fœtales circulantes ou à des expositions auto-antigéniques sous l'effet de la tension cutanée induite.

La biopsie cutanée révèlera un infiltrat cellulaire dermique constitué de lymphocytes et de polynucléaires éosinophiles associés à une spongiose et une parakératose avec un œdème dermique et de possibles dépôts de mucine. L'examen en immunofluorescence directe d'une biopsie cutanée peut montrer des dépôts granulaires de C3, d'IgM ou d'IgA à la jonction dermo-épidermique. II n'y a pas d'anticorps anti-membrane basale circulants. Ces deux dernières caractéristiques la différencient de la pemphigoïde gravidique, de pronostic plus sévère.

\section{Le prurigo actinique}

Le prurigo actinique est considéré comme une photodermatose très rare, à début précoce, en lien avec une susceptibilité génétique dépendant du système HLA (en particulier HLA DR4). Il existe une forme particulière aux amérindiens et des descriptions légèrement différentes et encore plus rares dans le nord de l'Europe [6], de lésions à type de prurigo débutant sur les zones photoexposées mais pouvant persister de façon perannuelle associées à une chéilite et une atteinte de la pointe du nez caractéristiques, avec une évolution cicatricielle. Une conjonctivite complète le tableau des formes amérindiennes tandis qu'une atopie est présente chez plus de la moitié des formes caucasiennes. Le diagnostic est fait par les tests photobiologiques en milieu spécialisé.

\section{Le prurigo pigmentosa}

Il s'agit d'une dermatose rare qui comporte des lésions papuleuses érythémateuses mais également des macules, des vésicules et un agencement réticulé avec une pigmentation réticulée séquellaire caractéristique, atteignant essentiellement le tronc. Initialement décrit chez des patients asiatiques, son étiopathogénie reste inconnue mais sa sensibilité à la dapsone et aux cyclines a été largement rapportée [7]. 


\section{Les examens paracliniques ont un double objectif}

\section{D'abord rechercher une néoplasie, une immunodépression ou une autre maladie sous-jacentes}

Les maladies sous-jacentes à un prurigo peuvent être une hémopathie (maladie de Vaquez, lymphome de Hodgkin, autres leucémies, anémies), une insuffisance rénale ou une cholestase, des carences vitaminiques primaires ou secondaires, une dysthyroïdie, un diabète, $d^{\prime}$ autres néoplasies solides.

Le bilan d'un prurigo, comme celui d'un prurit sine materia, comportera une numérationformule sanguine, la recherche d'un syndrome inflammatoire biologique, une électrophorèse des protéines sériques, un bilan hépatique et rénal et une glycémie à jeun, un bilan phosphocalcique et martial, une TSH, des sérologies du VIH et des hépatites virales. Sur point d'appel et selon l'anamnèse pourront être recherchées d'autres carences vitaminiques, diverses recherches parasitaires y compris la toxocarose. Une imagerie thoracique et abdominale peut être proposée.

\section{Puis écarter les diagnostics différentiels}

Le recours à une histopathologie avec immunofluorescence directe permettra d'écarter les diagnostics différentiels nombreux et pour certains difficiles : pemphigoïde bulleuse débutant par un prurigo, lichen plan hypertrophique, kérato-acanthomes éruptifs, dermatoses perforantes éruptives, nodules scabieux, etc.

\section{Conclusion}

Les prurigos chroniques sont des dermatoses aux contours flous mais dont la physiopathologie s'éclaire avec les avancées dans l'exploration des anomalies neurologiques fines retrouvées dans les lésions cutanées, ouvrant ainsi la voie à de nouvelles approches thérapeutiques.

\section{Références}

1. Pereira MP, Steinke S, Zeidler C, Forner C, Riepe C, Augustin M, et al. European academy of dermatology and venereology European prurigo project: expert consensus on the definition, classification and terminology of chronic prurigo. J Eur Acad Dermatol Venereol 2018;32:1059-65.

2. Zeidler C, Tsianakas A, Pereira M, Ständer H, Yosipovitch G, Ständer S. Chronic Prurigo of Nodular Type: A Review. Acta Derm Venereol 2018;98:173-9

3. Ohanyan T, Schoepke N, Eirefelt S, Hoey G, Koopmann W, Hawro T, et al. Role of Substance $P$ and Its Receptor Neurokinin 1 in Chronic Prurigo: A Randomized, Proof-ofConcept, Controlled Trial with Topical Aprepitant. Acta Derm Venereol 2018;98:26-31

4. Thijs JL, Strickland I, Bruijnzeel-Koomen CAFM, Nierkens S, Giovannone B, Csomor E, et al. Moving toward endotypes in atopic dermatitis: Identification of patient clusters based on serum biomarker analysis. J Allergy Clin Immunol 2017;140:730-737

5. Taylor D, Pappo E, Aronson IK. Polymorphic eruption of pregnancy. Clin Dermatol 2016;34:383-91 
6. Macfarlane L, Hawkey S, Naasan H, Ibbotson S. Characteristics of actinic prurigo in Scotland: 24 cases seen between 2001 and 2015. Br J Dermatol 2016;174:1411-4

7. Beutler BD, Cohen PR, Lee RA. Prurigo Pigmentosa: Literature Review. Am J Clin Dermatol 2015;16:533-43. 